\title{
Ultrahigh charge carrier mobility in nanotube encapsulated coronene stack
}

\author{
Saientan Bag and Prabal K. Maiti* \\ Center for Condensed Matter Theory, Department of Physics, Indian Institute of Science, Bangalore-560012, India
}

(Received 19 February 2017; published 4 December 2017)

\begin{abstract}
Achieving high charge-carrier mobility is the holy grail of organic electronics. In this paper we report an ultrahigh charge-carrier mobility of $14.93 \mathrm{~cm}^{2} \mathrm{~V}^{-1} \mathrm{~s}^{-1}$ through a coronene stack encapsulated in a singlewalled carbon nanotube (CNT) by using a multiscale modeling technique which combines molecular dynamics simulations, first-principle calculations, and kinetic Monte Carlo simulations. For a CNT having a diameter of $1.56 \mathrm{~nm}$ we find a highly ordered defect-free organization of coronene molecules inside the CNT which is responsible for the high charge-carrier mobility. The encapsulated coronene molecules are correlated with a large correlation length of $\sim 18 \AA$, which is independent of the length of the coronene column. Our simulation further suggests that coronene molecules can spontaneously enter the CNT, suggesting that the encapsulation is experimentally realizable.
\end{abstract}

DOI: 10.1103/PhysRevB.96.245401

\section{INTRODUCTION}

The successful use of organic electronics in everyday applications rely on the availability of materials with high charge-carrier mobility. For this purpose, there is a worldwide effort to design new materials that allow the fast movement of charge carriers through them. The highest charge-carrier mobility values reported so far in the literature lie in the range of $10-20 \mathrm{~cm}^{2} \mathrm{~V}^{-1} \mathrm{~s}^{-1}$ [1-4]. However the generation of morphology to achieve such high mobility requires sophisticated experimental procedures which are still very expensive and inefficient for large-scale production.

Use of flat disk-like molecules (discotic) which are often self-assembled in a columnar stack to form various discotic liquid-crystalline phases is an attractive route to device charge transporting materials in organic electronics [5]. Utilizing the high $\pi-\pi$ overlap [6-8] of the electronic cloud of discotics in a columnar arrangement, one-dimensional transport of charge through the stack is plausible. However, the efficiency of charge transport through the columnar arrangement of the discotics depends on the positional and orientational correlation of the discotics and is limited by the structural defects present in a column. To maximize charge-carrier mobility in a discotic assembly, two difficulties have to be overcome: First, one has to make the columnar arrangements defect free, and, second, one has to achieve optimum positional and orientational correlations between the molecules in the column. In this race to achieve high mobility in discotics, researchers have made significant progress in achieving a required correlation between the discotics in the column by tuning the core and tails of the discotics. Andrienko et al. [8] proposed a discotic material with triangular core and alternating hydrophilic-hydrophobic side chains which selfassemble in a discotic columnar phase with an average twist of $60^{\circ}$ between the molecules. With this particular discotic phase, they were able to attain a mobility of only $0.2 \mathrm{~cm}^{2} \mathrm{~V}^{-1} \mathrm{~s}^{-1}$ due to structural defects. So the main challenge at this stage is to find a way to make a defect-free organization of the molecules. Although the emergence of defects in the

*maiti@physics.iisc.ernet.in self-assembled discotics are inevitable due to the thermal fluctuation, the appearance of defects can significantly be reduced by suitable confinement [9-11]. In this paper we propose a way to achieve defect-free columnar aggregates and demonstrate ultrahigh charge-carrier mobility through the column. In particular we show defect-free columnar aggregates of coronene molecules inside a carbon nanotube (CNT). While this system had been previously studied by various groups $[12,13]$ in some different contexts, the charge transport properties of the confined coronene column has not been studied so far. In a recent experiment, Yoshida et al. [14] reported very high electrical conductivity in a well-stacked cationic coronene assembly prepared by electrochemical hole doping. We use molecular dynamics (MD) simulations to study the column formation inside CNTs of various diameter and report a critical value of the diameter of the CNT to achieve a highly ordered defect-free coronene stack. We use Marcus-Hush [15] formalism to calculate the charge-carrier mobility along the columnar stack and find an ultrahigh mobility of $14.93 \mathrm{~cm}^{2} \mathrm{~V}^{-1} \mathrm{~s}^{-1}$ at an electric-field strength of $10^{7} \mathrm{~V} / \mathrm{m}$ and a temperature of $300 \mathrm{~K}$. The calculated mobility value is two orders of magnitude higher than the mobility for discotic [8] systems reported so far in the literature. As we have already mentioned, the highest charge-carrier mobility values reported in some polymer semiconductor-based organic field-effect transistors (OFETs) [1-4] lie in the range of $\sim 10-20 \mathrm{~cm}^{2} \mathrm{~V}^{-1} \mathrm{~s}^{-1}$. The mobility value that we report in this paper is as high as these. It is worth mentioning that the CNT which acts as a template for the self-assembly of the coronene has much higher conductivity than the coronene stack. But the purpose of our model is to achieve high mobility in molecular systems such as coronene which has suitable optoelectronic and charge transport properties [14,16,17] for molecular electronics applications. In addition, we show that, by changing the diameter of the CNT, one can move from highly ordered to extreme disordered packing of coronene, which leads from ultrahigh mobility to almost zero hole mobility. Thus, our model also gives the tunability in the mobility which is often required in various applications.

We also perform a simulation where the coronene molecules are initially placed outside the nanotube. It shows the spontaneous insertion of the coronene molecules inside the CNT 
and the formation of a well-ordered column. This simulation suggests an experiment protocol where the CNT is placed inside the coronene vapor to achieve the CNT encapsulated coronene stack.

This paper is divided in three sections. In the first section we describe the MD simulation of the coronene stack. In the second section we describe the charge transport simulation and the results. Finally we end with the concluding remarks.

\section{MD SIMULATION}

A zigzag carbon nanotube with various radii was initially prepared by using Visual Molecular Dynamics (VMD) [18]. The coronene molecule was built and geometry optimized in GAUSSIAN 09 [19] by using the B3LYP/6-311G level of theory. The charge in the coronene molecules was calculated following the restrained electrostatic potential (RESP) method [20] using the GAUSSIAN 09 [19] and AMBERTOOLS [21] package. No charge was assigned to the carbon atom of the nanotube. Initially, 14 coronene molecules were arranged inside the nanotube in a columnar stack with a distance of $4 \AA$ between two neighboring molecules, as shown in Fig. 1(a). We prepared a series of systems as described above with CNT diameter ranging from 1.40 to $1.72 \mathrm{~nm}$. After initial energy minimization, systems were gradually heated from 0 to $300 \mathrm{~K}$. A production run of $50 \mathrm{~ns}$ was then performed in the NVT ensemble. During the simulation, the CNT was held fixed with its axis along the $z$ direction. All molecular dynamics simulations were performed by using the AMBER package [21] with the GAFF [22] force field. Equilibrated simulation snapshots of the systems are shown in Fig. 1(b). For a nanotube diameter between $1.40(n=18, m=0)$ and $1.56 \mathrm{~nm}(n=20, m=0)$ the coronene molecules maintain a well-ordered columnar structure inside CNT. For a diameter larger than that, no well-defined column forms inside the CNT [see Fig. 1(b) and Fig. S6 of the Supplemental Material [23]].

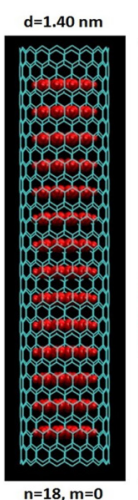

(a)

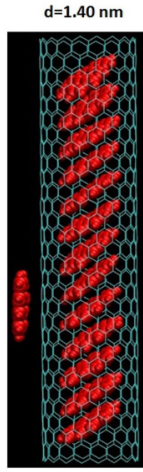

$n=18, m=0$

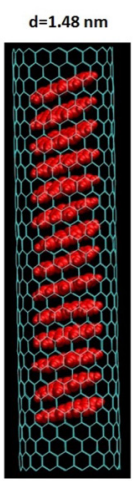

$n=19, m=0$

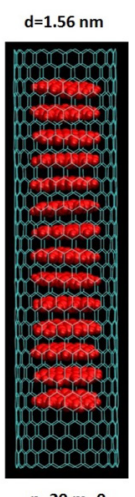

(b)

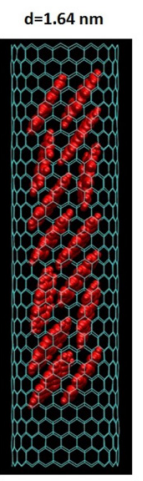

$n=21, m=0$

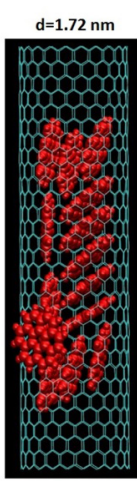

$\mathrm{n}=22, \mathrm{~m}=0$
FIG. 1. (a) Snapshot of the initial arrangement of the coronene molecules inside the CNT ( $n=18, m=0$ ). (b) Equilibrated simulation snapshot of the CNT encapsulated coronene system for the CNTs of diameter ranging from 1.4 to $1.72 \mathrm{~nm}$. For the CNTs with diameter between 1.40 and $1.56 \mathrm{~nm}$, a nice coronene column exists inside the nanotube. For larger diameter, no well-ordered column forms inside the CNT.

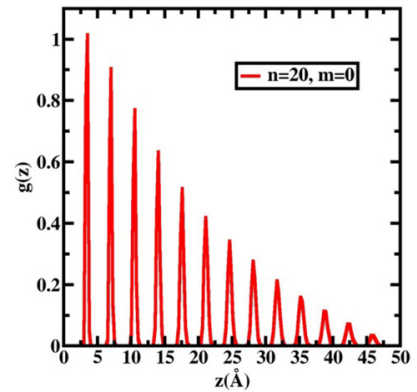

(a)

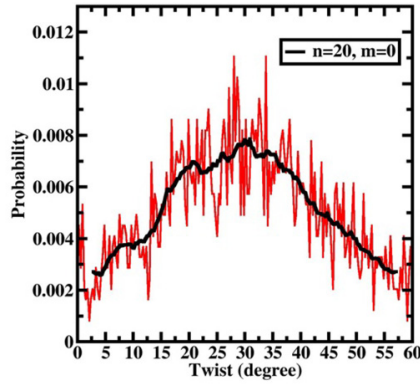

(b)
FIG. 2. (a) The pair correlation function of the coronene molecules and (b) twist between neighboring coronene molecules encapsulated inside a $(20,0)$ CNT. The sharp peaks in the correlation function in panel (a) indicate almost-defect-free arrangements of the coronene molecules inside the CNT. (b) The molecules prefer to have a twist angle of $30^{\circ}$ between them. The original data are shown in red while running-averaged data in black are shown in panel (b) to guide the eye.

Please note that, in bulk, the coronene molecules do not form any thermodynamically stable ordered structure [24].

Next we measure the positional and orientational correlation between the molecules stacked inside the CNT, since it determines the efficiency of charge transport through the coronene stack by controlling the $\pi-\pi$ electronic overlap between the molecules. The pair correlation function of the coronene molecules stacked inside the nanotube was calculated by using

$$
g(z)=\frac{1}{N} \sum_{i=1}^{N} \sum_{j=1, j \neq i}^{N}\left\langle\delta\left(z-z_{i j}\right)\right\rangle,
$$

where $z_{i j}$ is the axial separation between the $i$ th and the $j$ th coronene molecules, $N$ is the number of coronene molecules inside the nanotube, and the angular brackets indicate an average over time. The resultant plot for the $(20,0)$ CNT case is shown in Fig. 2(a). The pair correlation function for other cases is shown in Fig. S1 of the Supplemental Material [23]. The sharp peaks in the correlation function indicate almostdefect-free arrangements of the coronene molecules inside the nanotube. The average distance between two neighboring coronene molecules is $3.6 \AA$ for a $(20,0)$ CNT with a diameter of $1.56 \mathrm{~nm}$. Since the $\pi-\pi$ overlap between two coronene molecules decreases exponentially with the distance [8], the charge transport through the coronene column encapsulated in the $(20,0)$ CNT is expected to be most efficient.

At this point we ask whether the correlation between the coronene molecules as described above depends on the length of the column inside the nanotube. To answer this question, we simulate a long coronene stack of 42 molecules inside the $(20,0)$ CNT. We measure the pair correlation by using the same equation (1), but taking only the 14 molecules in the central region of the CNT to compare this with the correlation of the coronene molecules in the smaller stack (14 molecules). We found (see Fig. S2 of the Supplemental Material [23]) identical correlation among the coronene molecules for both cases. We further fit the correlation function to an exponential distribution (see Fig. S3 of the Supplemental Material [23]) and find a correlation length of $18 \AA$ between the coronene 
TABLE I. Structural and charge transport properties of a coronene column formed inside CNTs of different diameters.

\begin{tabular}{|c|c|c|c|c|c|c|}
\hline $\begin{array}{l}\text { CNT } \\
\text { chirality }\end{array}$ & $\begin{array}{l}\text { CNT } \\
\text { diameter } \\
(\mathrm{nm})\end{array}$ & $\begin{array}{c}\text { Average distance between } \\
\text { coronene molecules } \\
(\AA)\end{array}$ & $\begin{array}{l}\text { Average twist between } \\
\text { coronene molecules } \\
\text { (degrees) }\end{array}$ & $\begin{array}{c}\text { Average tilt of } \\
\text { coronene molecules } \\
\text { (degrees) }\end{array}$ & $\begin{array}{c}\text { Average } \\
\text { transfer integral } \\
(\mathrm{eV})\end{array}$ & $\begin{array}{c}\text { Hole } \\
\text { mobility } \\
\left(\mathrm{cm}^{2} \mathrm{~V}^{-1} \mathrm{~s}^{-1}\right)\end{array}$ \\
\hline$n=18, m=0$ & 1.40 & 4.30 & None & 35 & 0.080 & $4.21 \pm 0.17$ \\
\hline$n=19, m=0$ & 1.48 & 3.80 & None & 20 & 0.099 & $4.52 \pm 0.34$ \\
\hline$n=20, m=0$ & 1.56 & 3.60 & 30 & 4 & 0.172 & $14.93 \pm 0.49$ \\
\hline$n=11, m=11$ & 1.49 & 3.65 & None & 18 & 0.109 & $6.49 \pm 0.49$ \\
\hline$n=5, m=17$ & 1.56 & 3.60 & 30 & 4 & 0.159 & $14.41 \pm 0.57$ \\
\hline
\end{tabular}

molecules. We also calculate the twist between neighboring molecules in the column [Fig. 2(b)], since the overlap between the $\pi-\pi$ electronic cloud of the neighboring molecules strongly depends on the twist [8]. Average twist was found to be $30^{\circ}$ between the molecules for the system with CNT diameter of $1.56 \mathrm{~nm}$. To understand why the molecules prefer to have a $30^{\circ}$ twist, we calculated the energy (using the M06-2X/6-311G level of theory) of a pair of coronene as a function of the twist angle between them [see Fig. 4(a)]. The energy curve has a minimum at an angle of $30^{\circ}$, which explains the tendency of the molecule to achieve $30^{\circ}$ twist. However, there are no specific twists found (see Fig. S4 of the Supplemental Material [23]) between the coronene molecules confined in $(18,0)$ and $(19,0)$ CNTs. In these cases due to the smaller radius of the CNT the coronene molecules are tilted to accommodate themselves inside the CNT and do not have the rotational degree of freedom to reach to the state with a $30^{\circ}$ twist. We also measured the tilt of the molecules with respect to the axis of the CNT (see Fig. S5 of the Supplemental Material [23]) which completes the description of the arrangement of coronene molecules inside the CNT. Table I tabulates numerical values of all the quantities describing the arrangement of the coronene molecules inside the CNTs of various diameter and chirality.

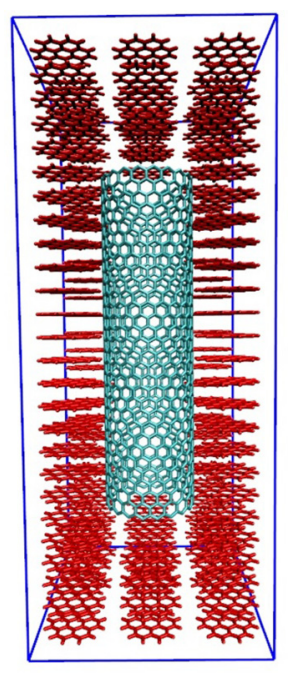

(a)

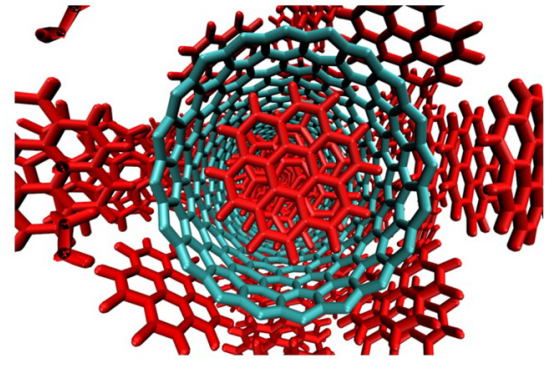

(b)
FIG. 3. (a) Initial arrangement of the system with a CNT placed in a box of coronene molecules. (b) Equilibrated snapshot of the system. Coronene molecules are spontaneously inserted into the nanotube and form columns.
Now we ask how one can achieve such a system in the laboratory. We perform another simulation with a CNT placed in a box of coronene molecules either in liquid or gas phase, as shown in Fig. 3(a). The coronene molecules spontaneously enter into the CNT and form a well-ordered column [Fig. 3(b)] within a few picoseconds of the simulation run. In this case also we followed the same simulation methodology as described in the previous paragraph. The two different systems, one with the coronene molecules outside CNT and the other with coronene molecules arranged inside the CNT, lead to same arrangements of the molecules after equilibration. This simulation suggests an experimental setup in which a CNT is placed in coronene vapor to achieve nanotube encapsulated coronene stacks.

To further validate our model from the experimental point of view, we performed another simulation where the CNT and the coronene molecules are solvated in the organic solvent THF (see Fig. S7 in the Supplemental Material [23]). In this case the coronene molecules also migrate and form ordered structures inside the CNT (see Fig. S8 in the Supplemental Material [23]).

\section{CHARGE TRANSPORT SIMULATION}

We now calculate the charge-carrier mobility through the columnar stack of coronene encapsulated inside CNTs, as obtained from the MD simulations. To calculate the mobility we use the semiclassical Marcus-Hush formalism [15] which has been extensively used in the past in various types of organic electronic materials, including discotics. In the framework of Marcus-Hush formalism charge transport is described as the thermally activated hopping mechanism between hopping sites $i$ and $j$ with rate

$$
\omega_{i j}=\frac{\left|J_{i j}\right|^{2}}{\hbar} \sqrt{\frac{\pi}{\lambda k T}} \exp \left[-\frac{\left(\Delta G_{i j}-\lambda\right)^{2}}{4 \lambda k T}\right] .
$$

Here, $J_{i j}$ is the transfer integral defined as $J_{i j}=\left\langle\Phi_{i}|\hat{H}| \Phi_{j}\right\rangle$. $\Phi_{i}$ and $\Phi_{j}$ are the diabatic wave function localized on the $i$ th and $j$ th hopping site, respectively. $\Delta G_{i j}$ is the free-energy difference between the hopping sites. $\lambda$ is the reorganization energy of charge transfer, $k$ is the Boltzmann constant, and $T$ is the temperature. In our case, charge hopping sites are the coronene molecules itself. So we shall use the terms "hopping site" and "coronene molecule" interchangeably. The highest occupied molecular orbital (HOMO) and the lowest unoccupied molecular orbital (LUMO) were taken as diabatic wave functions to calculate the transfer 
integral $J_{i j}$ for hole and electron transport, respectively. Since the HOMO and LUMO orbital of the coronene molecule is degenerate due to the molecular symmetry of coronene, we calculate four terms: $\left\langle\mathrm{HOMO}^{i}|\hat{H}| \mathrm{HOMO}^{j}\right\rangle$, $\left\langle(\mathrm{HOMO}-1)^{i}|\hat{H}| \mathrm{HOMO}^{j}\right\rangle, \quad\left\langle\mathrm{HOMO}^{i}|\hat{H}|(\mathrm{HOMO}-1)^{j}\right\rangle$, and $\left\langle(\mathrm{HOMO}-1)^{i}|\hat{H}|(\mathrm{HOMO}-1)^{j}\right\rangle . J_{i j}$ (for hole transport) was taken as the root mean square of these four terms. To calculate these terms we performed density functional theory calculations (at the level of B3LYP/6-311G) using GAUSSIAN 09 [19] along with the code available in the VOTCA-CTP module [25]. For an application of an electric field $F$ along the CNT axis the free-energy difference $\Delta G_{i j}$ turned out to be $F \cdot d_{i j}$. Here $d_{i j}$ is the displacement vector between the center of mass of two coronene molecules. The reorganization $\lambda$ was calculated by using

$$
\lambda=U^{n C}-U^{n N}+U^{c N}-U^{c C} .
$$

Here, $U^{n C}\left(U^{c N}\right)$ is the internal energy of the neutral (charged) coronene molecule with charged-state (neutral-state) geometry and $U^{n N}\left(U^{c C}\right)$ is the internal energy of the neutral (charged) molecule with neutral-state (charged-state) geometry. DFT calculations using the B3LYP/6-311G level of theory were performed to calculate all these energy terms in Eq. (3). Once all the rates $\omega$ between all the neighboring coronene molecules were calculated, then a kinetic Monte Carlo (KMC) simulation was done to simulate the transport of charge, and the charge mobility was predicted. The charge transport simulation was repeated with 30 different morphologies of the coronene column, as obtained from the MD simulation.

More details of the mobility calculation using the MarcusHush formalism can be found in the Refs. [6,7,25-27]. We find a hole mobility of $14.93 \mathrm{~cm}^{2} \mathrm{~V}^{-1} \mathrm{~s}^{-1}$ at an electric field of $10^{7} \mathrm{~V} / \mathrm{m}$ (applied along the CNT axis) and at a temperature of $300 \mathrm{~K}$ for the coronene column encapsulated inside a CNT of diameter $1.56 \mathrm{~nm}(n=20, m=0)$. For the systems with CNT diameters of 1.40 and $1.48 \mathrm{~nm}$, the mobility values were found to be 4.21 and $4.52 \mathrm{~cm}^{2} \mathrm{~V}^{-1} \mathrm{~s}^{-1}$, respectively. As mentioned in the previous section, for CNTs with a diameter larger than $1.56 \mathrm{~nm}$, there is no well-defined column formed inside the CNT. As a consequence, the hole mobility also drops to zero beyond that diameter. The highest mobility $\left(14.93 \mathrm{~cm}^{2} \mathrm{~V}^{-1} \mathrm{~s}^{-1}\right)$ that we report here is two orders of magnitude higher than the reported mobility values in discotics [8]. The reason behind such a dramatically high mobility lies in the form of the pair correlation function itself. The sharply peaked correlation function indicates solid-like ordering of the molecule inside the nanotube, which gives rise to a very sharply peaked distribution of transfer integrals [Fig. 4(b)], resulting in the high value of mobility [6]. As the diameter of the CNT decreases from $1.56 \mathrm{~nm}$, the average value of the transfer integral also decreases, resulting in a reduction in the mobility. The average values of the transfer integral and the mobility are tabulated in Table I for CNTs of various diameter and chirality. It is worth mentioning that the average twist between the coronene molecules $\left(30^{\circ}\right)$ for the system with highest mobility is not the best one can get in terms of achieving the highest transfer integral between neighboring molecules. A similar arrangement of the coronene molecules but with a $60^{\circ}$ twist will lead to much higher mobility. So far

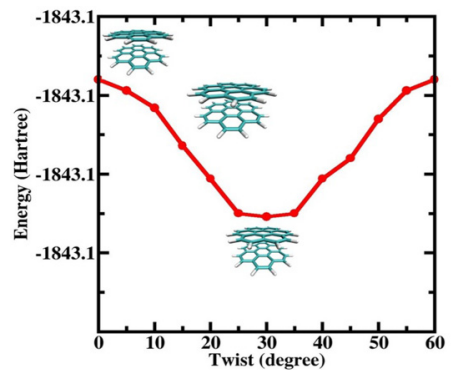

(a)

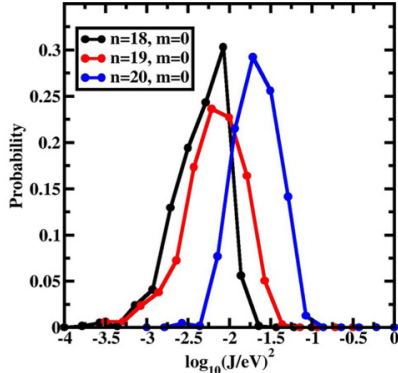

(b)
FIG. 4. (a) Energy of a pair of coronene molecules as a function of the twist angle between them. Snapshots of the coronene pair at different twist are shown in the inset of panel (a). (b) Probability distribution of the transfer integral between neighboring coronene molecules encapsulated inside the CNT.

we have not found a design principle to achieve such a twist for coronene.

So far we have talked about the encapsulation of coronenes in zigzag CNTs. However, there is nothing special about zigzag CNTs; it is the radius of the CNT that is important. A $(5,17)$ chiral CNT with the radius $1.56 \mathrm{~nm}$ will also gives rise to a similar arrangement (see Fig. S6 of the Supplemental Material [23]) of coronene inside the CNT and a charge-carrier mobility similar to that of the $(20,0) \mathrm{CNT}$ case. The possible armchair CNT with a radius close to the $1.56 \mathrm{~nm}$ are the $(11,11) \mathrm{CNT}$ with a diameter of $1.49 \mathrm{~nm}$ and the $(12,12)$ CNT with a radius of $1.63 \mathrm{~nm}$. None of these allow the best arrangement (see Fig. S6 of the Supplemental Material [23]) of the coronene inside themselves to achieve the ultrahigh mobility as found in the coronene column inside CNT of diameter $1.56 \mathrm{~nm}$.

\section{CONCLUSIONS}

Coronene molecules spontaneously self-assemble inside CNTs of different chirality. For a specific diameter of $1.56 \mathrm{~nm}$, which corresponds to either a $(20,0)$ zigzag or a $(5,17)$ chiral tube, we achieve solid-like ordering with an average spacing of 3.6 ̊ between the coronene molecules. In the confined state we see the crystal-like local positional ordering of the molecules which gives rise to a highly efficient charge transport pathway through the coronene column. We report a charge-carrier mobility of $14.93 \mathrm{~cm}^{2} \mathrm{~V}^{-1} \mathrm{~s}^{-1}$ in this system, which is two orders of magnitude higher than the reported mobility in the discotics so far [8]. We have also shown how the mobility can be tuned by changing the CNT diameter. We have given a proof-of-principle demonstration to achieve such ordering inside CNTs. The reported system should be easily achievable experimentally and successful use of it will lead it to a new frontier in energy applications.

\section{ACKNOWLEDGMENTS}

We acknowledge financial support from Department of Science and Technology (DST), India. We acknowledge Prof. K. Ganapathy Ayappa for careful reading of the manuscript. We thank Prof. Manish Jain for helpful discussions. 
[1] H. Minemawari, T. Yamada, H. Matsui, J. Y. Tsutsumi, S. Haas, R. Chiba, R. Kumai, and T. Hasegawa, Nature (London) 475, 364 (2011).

[2] Y. Diao, B. C. Tee, G. Giri, J. Xu, D. H. Kim, H. A. Becerril, R. M. Stoltenberg, T. H. Lee, G. Xue, S. C. Mannsfeld, and Z. Bao, Nat. Mater. 12, 665 (2013).

[3] H. R. Tseng, H. Phan, C. Luo, M. Wang, L. A. Perez, S. N. Patel, L. Ying, E. J. Kramer, T. Q. Nguyen, and G. C. Bazan, Adv. Mater. 26, 2993 (2014).

[4] I. L. Kang, H. J. Yun, D. S. Chung, S. K. Kwon, and Y. H. Kim, J. Am. Chem. Soc. 135, 14896 (2013).

[5] D. Adam, P. Schuhmacher, J. Simmerer, L. Häussling, K. Siemensmeyer, K. Etzbachi, H. Ringsdorf, and D. Haarer, Nature (London) 371, 141 (1994).

[6] S. Bag, V. Maingi, P. K. Maiti, J. Yelk, M. A. Glaser, D. M. Walba, and N. A. Clark, J. Chem. Phys. 143, 144505 (2015).

[7] J. Kirkpatrick, V. Marcon, J. Nelson, K. Kremer, and D. Andrienko, Phys. Rev. Lett. 98, 227402 (2007).

[8] X. Feng, V. Marcon, W. Pisula, M. R. Hansen, J. Kirkpatrick, F. Grozema, D. Andrienko, K. Kremer, and K. Mullen, Nat. Mater. 8, 421 (2009).

[9] R. Zhang, X. Zeng, B. Kim, R. J. Bushby, K. Shin, P. J. Baker, V. Percec, P. Leowanawat, and G. Ungar, ACS Nano 9, 1759 (2015).

[10] C. V. Cerclier, M. Ndao, R. M. Busselez, R. Lefort, E. Grelet, P. Huber, A. V. Kityk, L. Noirez, A. Schönhals, and D. Morineau, J. Phys. Chem. C 116, 18990 (2012).

[11] R. Busselez, C. V. Cerclier, M. Ndao, A. Ghoufi, R. Lefort, and D. Morineau, J. Chem. Phys. 141, 134902 (2014).

[12] B. Verberck, T. Okazaki, and N. Tarakina, Phys. Chem. Chem. Phys. 15, 18108 (2013).

[13] I. V. Anoshkin, A. V. Talyzin, A. G. Nasibulin, A. V. Krasheninnikov, H. Jiang, R. M. Nieminen, and E. I. Kauppinen, ChemPhysChem 15, 1660 (2014).
[14] Y. Yoshida, K. Isomura, H. Kishida, Y. Kumagai, M. Mizuno, M. Sakata, T. Koretsune, Y. Nakano, H. Yamochi, M. Maesato, and G. Saito, Chem. Eur. J. 22, 6023 (2016).

[15] R. A. Marcus, Rev. Mod. Phys. 65, 599 (1993).

[16] S. Sanyal, A. K. Manna, and S. K. Pati, J. Mater. Chem. C 2, 2918 (2014).

[17] T. Okazaki, Y. Iizumi, S. Okubo, H. Kataura, Z. Liu, K. Suenaga, Y. Tahara, M. Yudasaka, S. Okada, and S. Iijima, Angew. Chem., Int. Ed. 50, 4853 (2011).

[18] W. Humphrey, A. Dalke, and K. Schulten, J. Mol. Graphics 14, 33 (1996).

[19] M. J. Frisch et al., GAUSSIAN09, Revision A.02 (Gaussian, Inc., Wallingford CT, 2009).

[20] C. I. Bayly, P. Cieplak, W. Cornell, and P. A. Kollman, J. Phys. Chem. 97, 10269 (1993).

[21] D. A. Pearlman, D. A. Case, J. W. Caldwell, W. S. Ross, T. E. Cheatham, S. DeBolt, D. Ferguson, G. Seibel, and P. Kollman, Comput. Phys. Commun. 91, 1 (1995).

[22] J. Wang, R. M. Wolf, J. W. Caldwell, P. A. Kollman, and D. A. Case, J. Comput. Chem. 25, 1157 (2004).

[23] See Supplemental Material at http://link.aps.org/supplemental/ 10.1103/PhysRevB.96.245401 for additional data and analysis.

[24] A. I. Chernov, P. V. Fedotov, I. V. Anoshkin, A. G. Nasibulin, E. I. Kauppinen, V. L. Kuznetsov, and E. D. Obraztsova, Phys. Status Solidi B 251, 2372 (2014).

[25] V. Rühle, A. Lukyanov, F. May, M. Schrader, T. Vehoff, J. Kirkpatrick, B. Baumeier, and D. Andrienko, J. Chem. Theory Comput. 7, 3335 (2011).

[26] S. Bag, M. Jain, and P. K. Maiti, J. Phys. Chem. B 120, 9142 (2016).

[27] S. Bag, S. Mogurampelly, W. A. Goddard III, and P. K. Maiti Nanoscale 8, 16044 (2016). 